\title{
Intelligent Building Management Systems using Multi Agents: Fuzzy Approach
}

\author{
Hamid Reza Naji \\ Kerman Graduate University of \\ Technology, Kerman, Iran \\ International Center for Science, \\ High Technology \& Environmental \\ Sciences, Iran
}

\author{
Morteza Nabi Meybodi \\ MA Candidate of IT management \\ Tarbiat Modares University \\ Iran, Tehran
}

\author{
Taha Nejad Falatouri \\ Moghaddam \\ MA Candidate of IT management \\ Tarbiat Modares University \\ Iran, Tehran
}

\begin{abstract}
Growing needs of humanity have forced him to use modern technology in all aspects of his life. Intelligent buildings are one of these modern technologies that have great advantages for people. These buildings include a set of integrated and dynamic systems to manage the allocation of resources and facilitate the residents' lives. In this context and due to high speed, reliability and better respond to environmental changes, we simulate such environment using multi agents with fuzzy approach. To do this, we use Matlab and its fuzzy toolbox. The results shows that parallel processing nature in this kind of multi agents improve the speedup of the system.
\end{abstract}

\section{Keywords}

Intelligent building, multi agents, fuzzy model.

\section{INTRODUCTION}

According to the Intelligent Building Institution in Washington, intelligent building is defined as "one which integrates various systems to effectively manage resources in a coordinated mode to maximize: technical performance, investment and operating cost savings, flexibility" [1]. In recent years, a lot of activities have performed in this context. Nowadays, multi agent systems have used in the most industries widely [2-6].

Hagras et al present an approach to the implementation of IB agents based on a hierarchical fuzzy genetic multi-embeddedagent architecture. In their prototype system, each agent has six inputs made up of four environmental variables - a room temperature, the outside natural temperature, the room illumination and the outside natural illumination. The two remaining inputs to the system are the room occupancy flag and another input indicating whether there is an emergency alarm. The system has two outputs which are the room heating setting and a room illumination setting [7].

The purpose of this paper is to use multi agent and parallel processing concepts with intelligent buildings. To accomplish this purpose, this paper begins with the discussion of the concept of intelligent buildings and agents. Then, we continue with introducing BDI approach and parallel processing. In the next, we present the architecture of an office with agents and sensors and agent-based model of intelligent buildings. Furthermore, we present fuzzy model and sample codes of the system. Finally, we simulate the system and perform the comparison test between serial and parallel processing.

\section{INTELLIGENT BUILDINGS}

For many years, buildings that offer comfortable, flexible and energy efficient living environment at a minimal cost has been the expectation of building owners and occupiers. To attain this aspiration, a variety of advanced building technologies have been developed in the past two decades, aiming to improve the building performance to satisfy a variety of human needs and environmental sustainability [8]. In this regard, the subject of intelligent building was introduced. An intelligent building is one that utilizes computer technology to autonomously govern and adapt the building environment so as to optimize user comfort, energy-consumption, safety and work efficiency [7]. The word "intelligent" was first used to describe buildings in the United States at the beginning of the 1980s. The concept of 'intelligent building' was stimulated by the development of information technology. Research on intelligent building has been conducted ubiquitously and research results have been published in many academic journals [1].

Nowadays, all governments pay great attention to energy-saving issues. So the need for energy productivity has increased. Energy management is a necessary activity for energy productivity. Energy management is considered as a significant factor to the energy saving of an operational unit of production. The basic concept of energy management is the continuous, systematic and well-organized audit of energy consumption, aiming at energy cost optimization with respect to energy demands, user characteristics, funding opportunities, financing ability and emission reductions achieved [9]. Moreover, ten modules are presented in order to deal with the health issues for buildings. These modules includes: M1: environmental friendliness - health and energy conservation; M2: space utilization and flexibility; M3: cost effectiveness-operation and maintenance with emphasis on effectiveness; M4: human comfort; M5: working efficiency; M6: safety and security measures - fire, earthquake, disaster and structural damages, etc. M7: culture; M8: image of high technology; M9: construction process and structure; and M10: health and sanitation. Intelligent building is redefined as one which designed and constructed based on an appropriate selection of these Quality Environmental Modules to meet the user's requirements [1].

In recent years, a lot of technologies and activities have been performed to improve intelligent buildings. We can use modern 
technologies such as wireless communication and new methods such as fuzzy, genetic algorithm, expert systems and multi agent systems for better management of these buildings $[1,7,10,11]$.

Nowadays, multi agent systems are spreading among different systems for solving complex problems. Building industries are one of the industries that we can use multi agent systems there. In these systems, we have a lot of sensors and effectors that are distributed in the building and the task of agents is convergence and integrating receiving information to transmit proper signals to the central system [12]

\section{BDI APPROACH}

One of the main methods of applying multi agent systems are BDI approach. In this approach, each agent has a set of beliefs, desires and intentions. Beliefs include the information that exists in the main memory of system and are constantly being updated. Desires represent objectives and situations that an agent would like to accomplish or bring about and intentions represent what an agent is chosen to do.

In our case, the beliefs in light agent include initial values that are received from indoor and outdoor sensors. Desires can be standards that are represented by international associations. For example, the proposed luminance for workplaces is among 500, 750, 1000 lux [13]. Agents intend to process and integrate receiving information and transmit output to a central system (mother agent). In the central system, some analysis performed with the fuzzy approach. The results are transmitted to controllers as signals. Finally, controllers do necessary changes.

\section{AGENTS for PARALLEL PROCESSING}

To date, agents have always been implemented in software because of software's inherent flexibility and ease of implementation. The current state of Field Programmable Gate Array, FPGA, technology and other reconfigurable hardware makes it possible to enjoy much of the flexibility in hardware, which formerly was only available in software. Implementation of agent techniques in reconfigurable hardware allows for the creation of high-speed systems with extensive fine-grained parallelism [14]. The parallel nature of the reconfigurable hardware would cause speedup to be further increased if more than one agent were implemented in the reconfigurable hardware. It is believed that the use of agents may prove useful in a number of application domains, where speed, flexibility, and evolutionary design goals are important issues [15].

Parallel processing can be performed in different ways. You can see one sample for intelligent building in figure 1 .

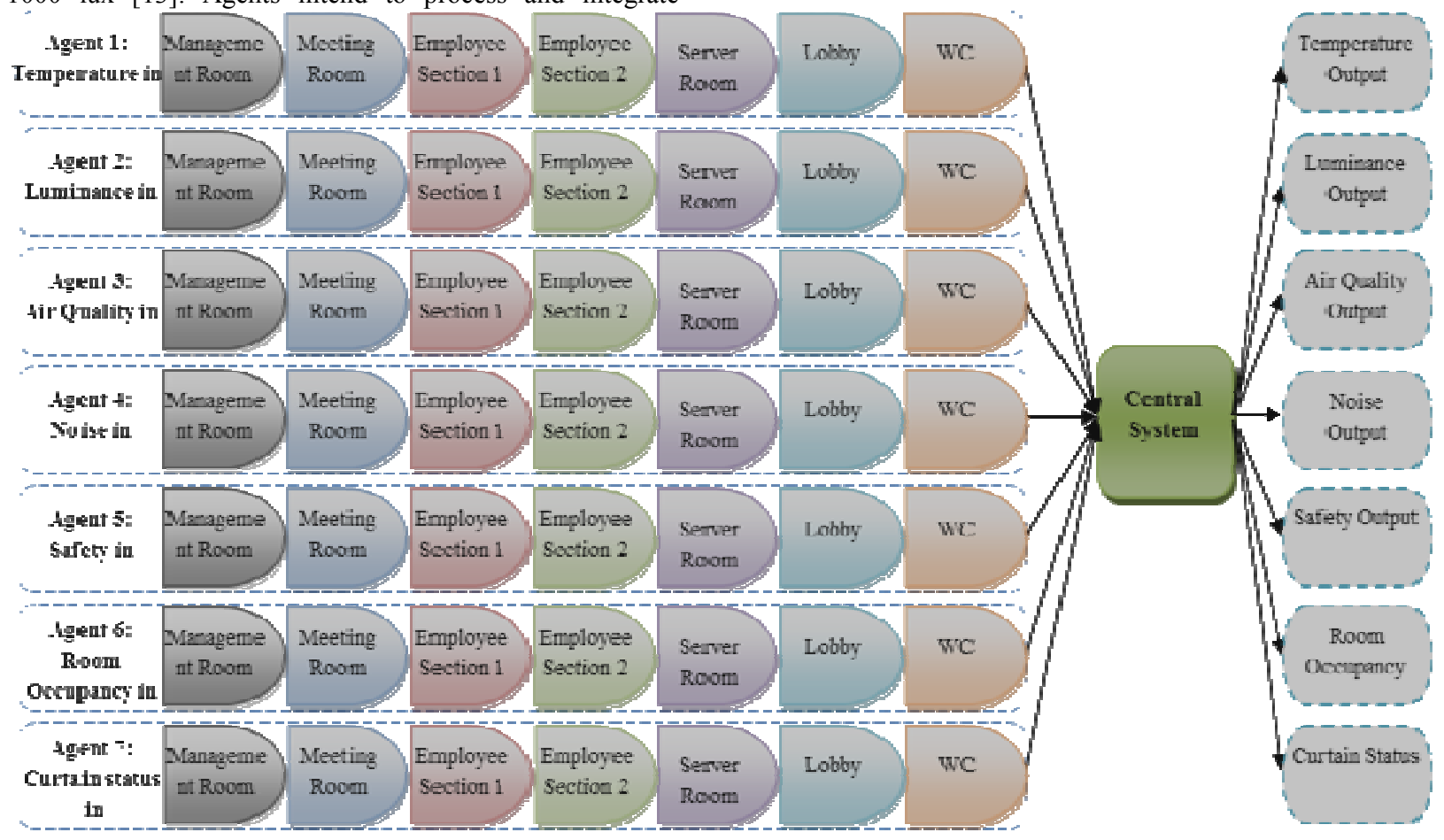

Figure 1: Parallel Processing

\section{INTELLIGENT BUILDING}

In figure 2, architecture of an office is drawn. In this figure, the place of agents and sensors is specified. Agents can be placed in server room.

Figure 3 shows an agent-based model of each section of building. For example, in management room, sensors such as light, temperature, air quality, noise, movement and ... can be placed inside the room or outside the building. Each sensor sends its information to the related agents. Agents process information and sent them to the fuzzy controller. Fuzzy controller transmits a proper signal to switches, valves and .... 


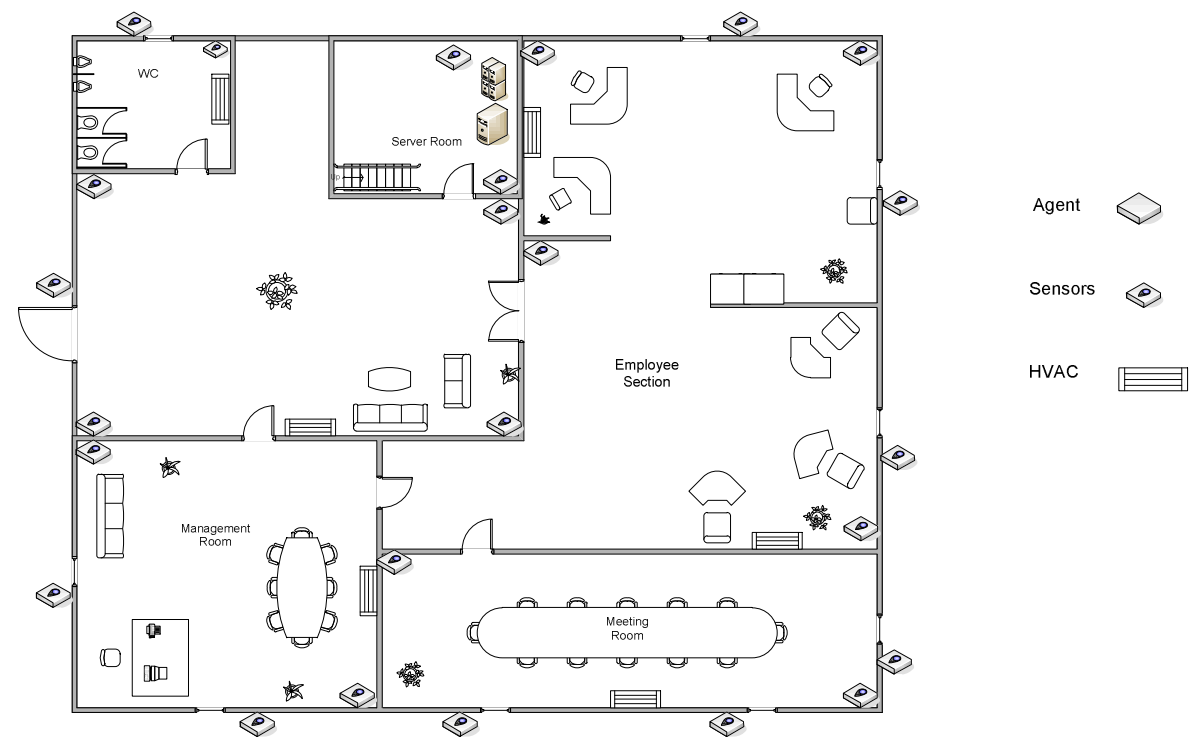

Figure 2: The architecture of an office with agents and sensors

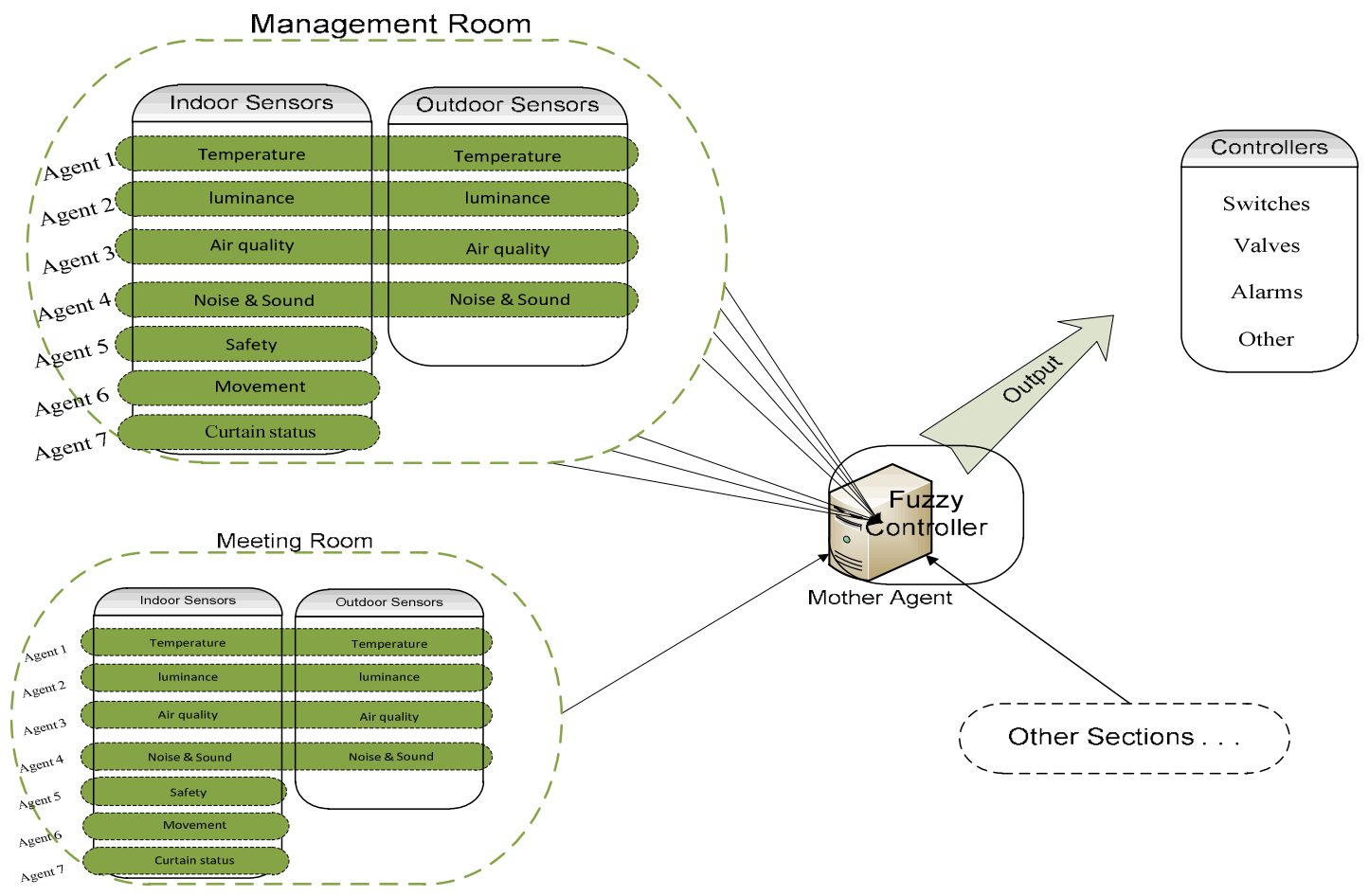

Figure 3: Agent-based model of intelligent building

\section{FUZZY MODEL}

Fuzzy logic offers a framework for representing imprecise, uncertain knowledge. Fuzzy systems use a mode of approximate reasoning, which allows it to deal with vague and incomplete information. In addition fuzzy controllers exhibit robustness with regard to noise and variations of system parameters [7]. Unlike crisp (or ordinary) sets, fuzzy sets have no sharp or precise boundaries. In crisp logic, every statement is true or false; i.e., it has a truth value 1 or 0 . In contrast, fuzzy sets have more flexible membership requirements that allow for partial membership in a set. Everything is a matter of degree, and exact reasoning is viewed as a limiting case of approximate reasoning [16]. We can easily use fuzzy with Matlab ${ }^{\mathrm{TM}}$ fuzzy toolbox.

For implementing fuzzy model, we should define required parameters in Matlab FIS toolbox. As a sample, we do this for luminance. 


\subsection{Fuzzy inference system for luminance}

First, we should identify affecting factors for luminance. These factors can be outdoor and indoor luminance, curtain status and room occupancy. Figure 4 shows these factors and their relations.

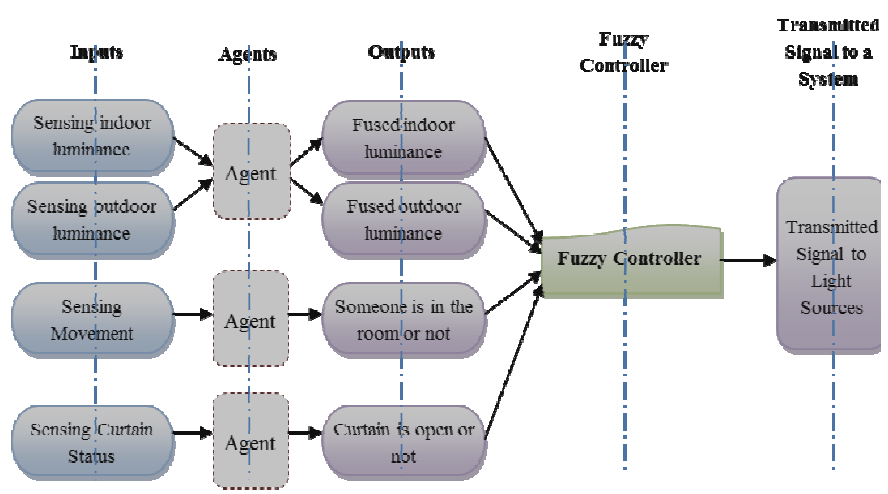

Figure 4: Fuzzy controller

In figure 5, we define fuzzy controller for luminance in Matlab.

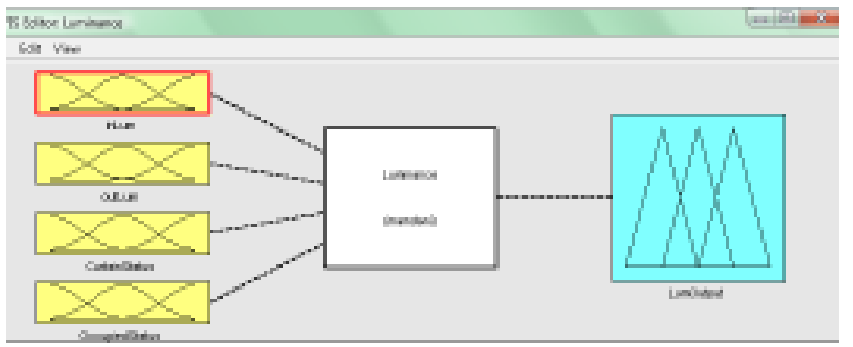

Figure 5: Fuzzy controller for luminance in Matlab

In figure 5, we have: inLum: indoor luminance, outLum: outdoor luminance, curtain status and occupied status.

Figures 6 to 8 show the membership functions of parameters.

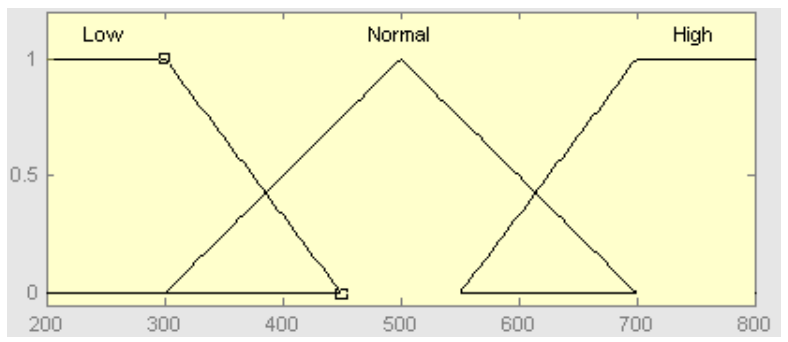

Figure 6: Membership function for indoor luminance

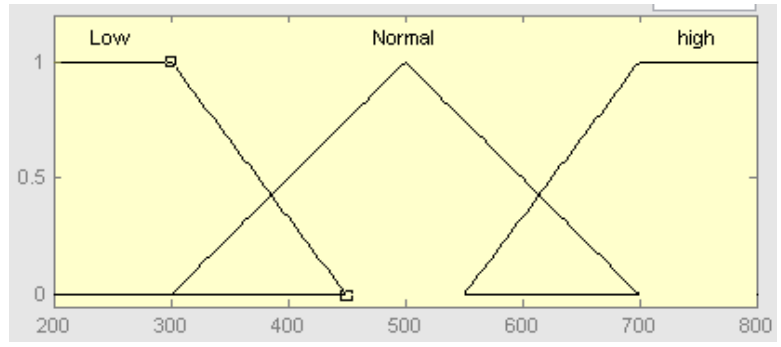

Figure 7: Membership function for outdoor luminance

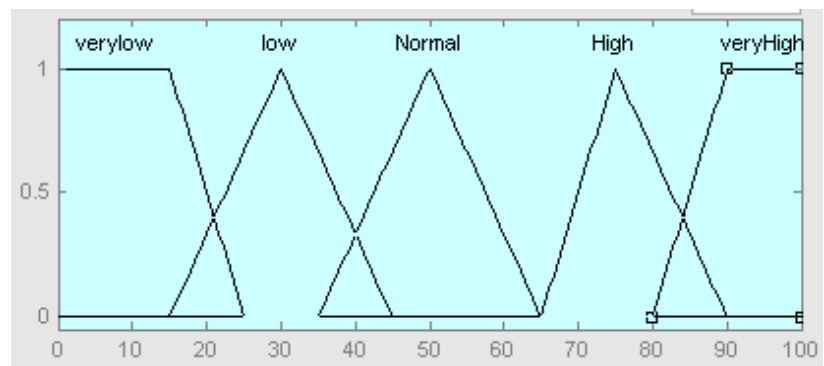

Figure 8: Membership function for output luminance (\%)

Curtain status and occupied status is defined in crisp (0 or 1). 1 means the curtain is open and a person is in the room.

Now, we can define our rules to determine the status of room luminance. An expert can help us in this regard. Considering different states that can be happened, some of the rules that can be defined are:

- If nobody was inside the room then change status to VeryLow

- If someone was inside the room and the curtain was closed then:

- If indoor luminance wasn't High then change status to High.

o If indoor luminance was High then don't change the status.

Figure 9 shows these rules in Matlab.

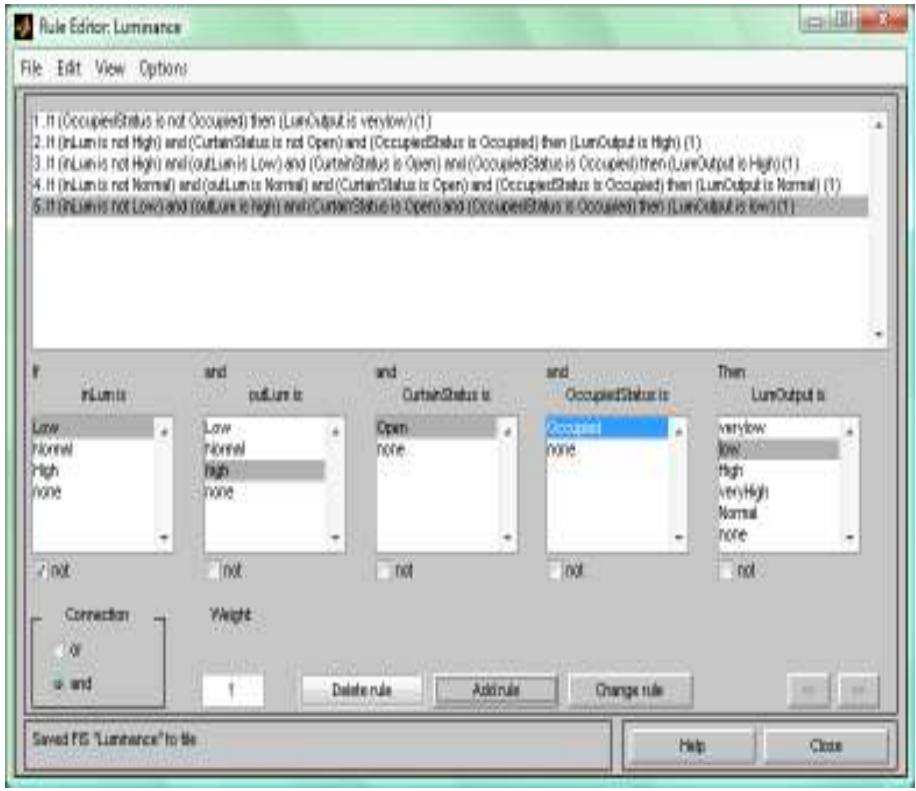

Figure 9: Rules for luminance in Matlab

Now, we can use these rules and data for defuzzification and get crisp results. You can see one sample in figure 10. 


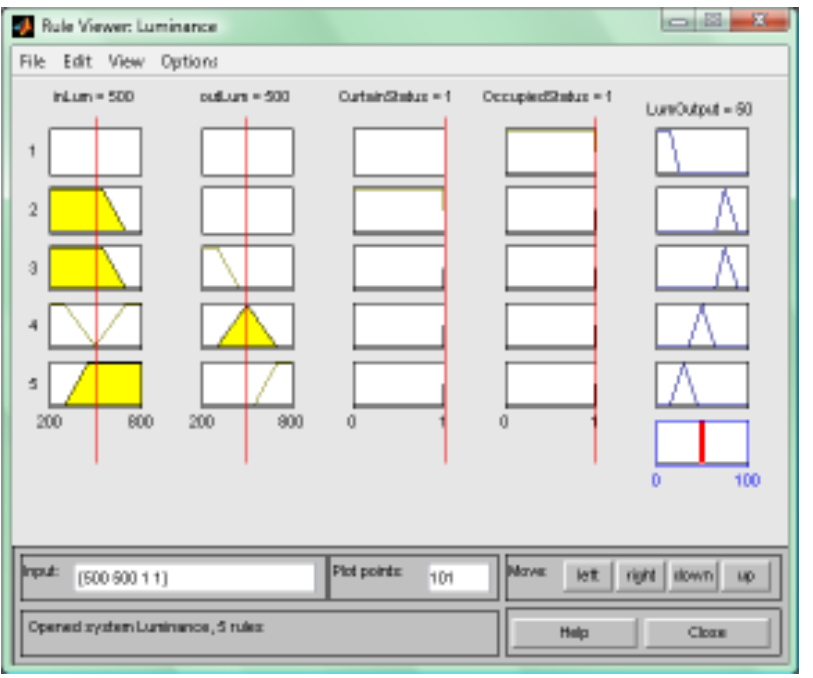

Figure 10: defuzzification in Matlab

In figure 10, we can see that if indoor luminance was 500 and outdoor luminance was 500 lux, someone be in the room and curtain be open then the room luminance status must be $50 \%$.

We can define room temperature like room luminance.

\section{NOISE, AIR QUAILTY and SAFETY}

Related standards for noise, air quality and safety have been defined by different institutions. These standards usually have specified critical points. We can set our application to alert us about these critical points. We can define these standards as ifthen rules. For example, if noise in the environment exceeds 55 $\mathrm{dB}$ in a daytime, then red alert starts.

We can find the related standards for noise, quality and safety from different sources in the internet and responsible institutions.

\section{SIMULATION RESULTS}

The system can be divided into two parts: Part related to the sensors and agents and part related to the fuzzy controller. In part 1 , agents get data from indoor and outdoor sensors and fuse them. Then, this information is sent to the fuzzy controller (part 2). Fuzzy controller calculates outputs (that are transmitted as signals to the controllers).

The following codes are written in Matlab for luminance in a sample office room. For simplicity, we use only two light sensors and arithmetic mean for fusion. For real cases, we can have much more sensors and use more complex methods for fusing data

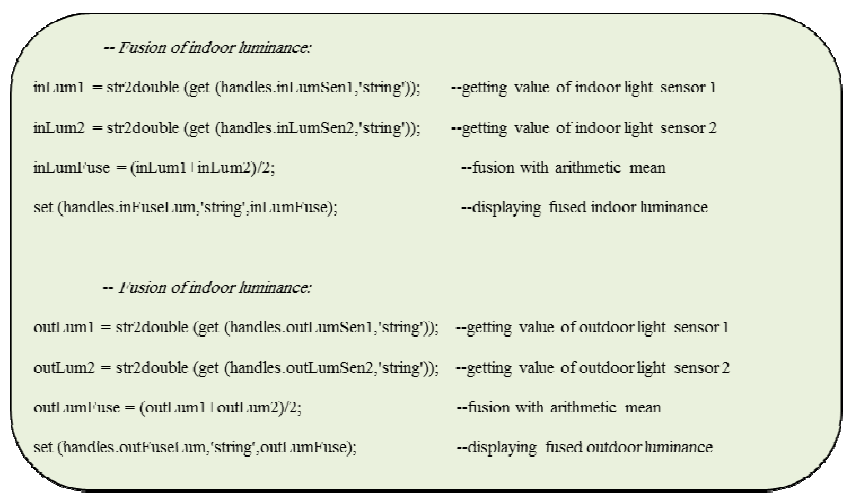

Figure 11: code segment 1- getting data and fuse them

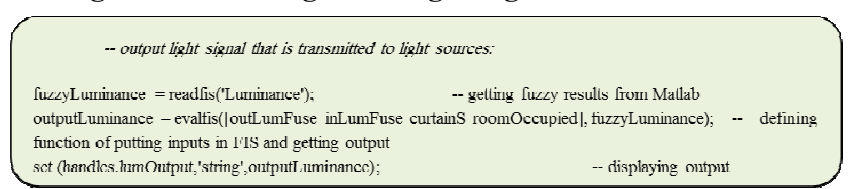

Figure 12: Inference in fuzzy controller

Figure 13 shows Application GUI with sample data.

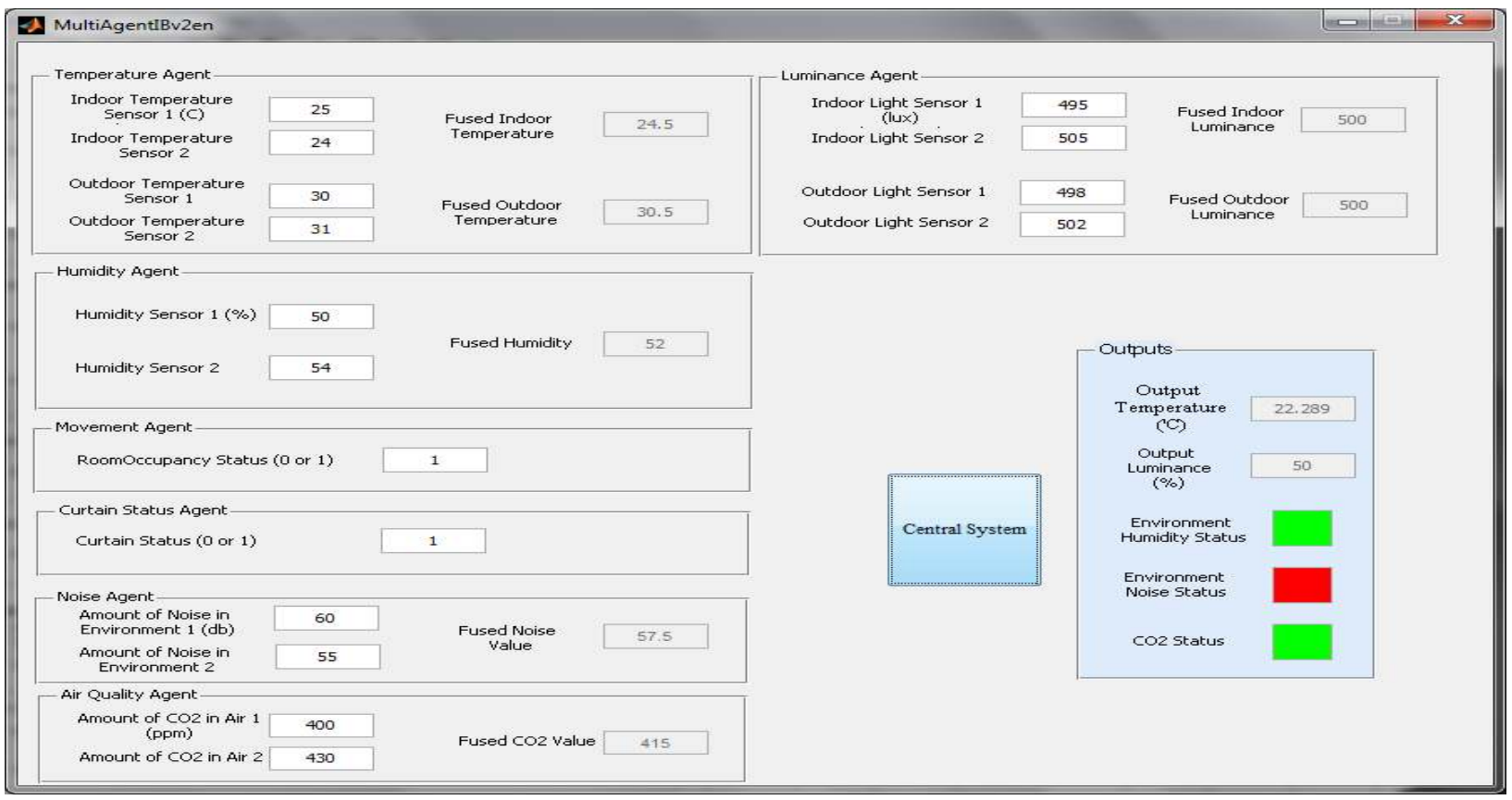

Figure 13: Application GUI with sample data 
We compare the results between serial and parallel processing (multi agents). This test is implemented in a computer with Intel Core 2 Due T5550 1.83 GHz CPU. Table 1 shows the results.

Table 1: comparison between parallel and serial processing

\begin{tabular}{|c|c|c|}
\hline $\begin{array}{c}\text { Runtime in serial } \\
\text { processing (average) }\end{array}$ & $\begin{array}{c}\text { Runtime in parallel } \\
\text { processing (average) }\end{array}$ & Speedup \\
\hline $10.657 \mathrm{~ms}$ & $2.183 \mathrm{~ms}$ & 5.03 \\
\hline
\end{tabular}

\section{CONCLUSION}

Generally, we can say that intelligent building management system is a set of software and hardware for monitoring and controlling different sections of a building. The goals of this system are balancing the work of various components of building and optimizing user comfort and energy-consumption. One of the methods to implement these systems is Multi agent systems with reconfigurable hardware. These kinds of systems can accelerate responses because of parallel processing. In this article, necessary frameworks for implementing these systems are presented. Moreover, we use fuzzy concepts to get more reliable results. We do this with Matlab fuzzy toolbox. The results show that parallel processing is faster than serial processing.

\section{REFERENCES}

[1] Wong, J.K.W., H. Li, and S.W. Wang. 2005, Intelligent building research: a review. Automation in Construction. 14: p. $143-159$.

[2] Ren, Z. and C.J. Anumba. 2004, Multi-agent systems in construction-state of the art and prospects. Automation in Construction. 13(3): p. 421-434.

[3] Rodin, V., et al. 2004, An immune oriented multi-agent system for biological image processing. Pattern Recognition. 37(4): p. 631-645.

[4] Guo, Q. and M. Zhang. 2009, A novel approach for multiagent-based Intelligent Manufacturing System. Information Sciences. 179(18): p. 3079-3090.

[5] Chen, K.-Y. and C.-J. Chen. 2010, Applying multi-agent technique in multi-section flexible manufacturing system. Expert Systems With Applications. 37(11): p. 7310-7318.
[6] Vengattaraman, T., et al. 2011, An application perspective evaluation of multi-agent system in versatile environments. Expert Systems With Applications. 38(3): p. 1405-1416.

[7] Hagras, H., et al. 2003, A hierarchical fuzzy-genetic multiagent architecture for intelligent buildings online learning, adaptation and control. Information Sciences. 150: p. 33-57.

[8] Wong, J.K.W. and H. Li. 2008, Application of the analytic hierarchy process (AHP) in multi-criteria analysis of the selection of intelligent building systems. Building and Environment. 43: p. 108-125.

[9] Doukas, H., C. Nychtis, and J. Psarras. 2009, Assessing energy-saving measures in buildings through an intelligent decision support model. Building and Environment. 44: p. 290- 298.

[10] Etik, N., et al. 2009, Fuzzy expert system design for operating room air-condition control systems. Expert Systems with Applications. 36: p. 9753-9758.

[11] Singh, J., N. Singh, and J.k. Sharma. 2006, Fuzzy modeling and control of HVAC system - A review. Journal of Scientific \& Industrial Research. 65: p. 470-476.

[12] Carrascosa, C., et al. 2008, Hybrid multi-agent architecture as a real-time problem-solving model Expert Systems with Applications. 34(1): p. 2-17.

[13] Neufert, E., et al. 2000, Architects' data: Blackwell Science.

[14] Naji, H.R., et al. 2002. Parallel Image Processing With Agent-based Reconfigurable Hardware. in 15th International Conference on Parallel and Distributed Computing Systems (PDCS 2002). Louisville, KY.

[15] Naji, H.R., B.E. Wells, and L. Etzkorn. 2004, Creating an Adaptive Embedded System by Applying Multi Agent Techniques to Reconfigurable Hardware Future Generation Computer Systems. 20(6): p. 1055-1081.

[16] Kahraman, C. 2008, Multi-criteria decision making methods and fuzzy sets. Springer Optimization and Its Applications. 16: p. $1-18$. 